\title{
EFFECT OF MAGNETIC POLARON FORMATION ON THE EXCITON MOBILITY EDGE IN $\mathrm{Cd}_{1-x} \mathrm{Mn}_{x} \mathrm{Te}$
}

\author{
G. Mackh, D.R. Yakovlev*, W. Ossau, A. WaAg and G. Landwehr
}

Physikalisches Institut der Universität Würzburg, 97074 Würzburg, Germany

We study the exciton localization in the semimagnetic semiconductor $\mathrm{Cd}_{1-x} \mathrm{Mn}_{x} \mathrm{Te}$ by selective excitation of the exciton photoluminescence. We show that the energy position of the effective mobility edge for excitons is subject to the competition between nonmagnetic and magnetic localization due to the magnetic polaron formation. External magnetic fields affect this competition by suppressing the polaron formation, which shifts the mobility edge.

PACS numbers: 78.55.Et

Effects of exciton localization in random potential fluctuations of mixed nonmagnetic semiconductors have been investigated by several authors $[1,2]$. Evidence has been found for a critical energy $E_{\text {em }}$, which separates the localized from the delocalized exciton states in the fluctuating band potential [1]: This critical energy $E_{\text {em }}$ is known as effective mobility edge (EME), above which exciton motion is possible within the decay time and below which excitons are localized and recombine without spatial migration. The energy position of the EME is determined by the characteristic times of spectral relaxation due to phonon emission. These characteristic times are a function of energy since the energy transfer to lower energy sites depends on the shape of the density of localized states [2]. The EME is experimentally elusive in nonmagnetic semiconductors. Evidence for an EME has been found from the exciton-one-LO-phonon band luminescence [1].

In semimagnetic semiconductors the excitons experience a nonmagnetic and a magnetic localization additionally $[3,4]$. The magnetic localization is induced by magnetic fluctuations and the magnetic polaron formation [5], which means the alignment of the spins of the magnetic ions in the vicinity of a localized carrier. The localized magnetic polaron (LMP) formation causes a low-energy shift of the photoluminescence (PL) line. Since the localized magnetic polaron formation is a fast process of the order of $100 \mathrm{ps}[5,6]$, it can interrupt the spatial diffusion of

* On leave from: A.F. Ioffe Physico-Technical Institute, Russian Academy of Sciences, 194021, St. Petersburg, Russia. 
excitons and thus hinder the nonmagnetic spectral relaxation to deep fluctuations of the band potential. The magnetic polaron formation is effectively suppressed in magnetic fields. Thus, the application of magnetic fields is expected to act on the competition between magnetic and nonmagnetic localization and on EME. The issue of this paper is to show that EME is subject to this competition.

For photoluminescence measurements the samples were inserted in pumped liquid helium $(1.8 \mathrm{~K})$ in the bore of a superconducting split-coil magnet. Magnetic fields were applied in the Faraday geometry parallel to the structure growth axis. Excitation was provided by an argon-ion pumped DCM dye laser. Spectra were taken with a $1 \mathrm{~m}$ spectrometer and a cooled photomultiplier with associated photon counting system.

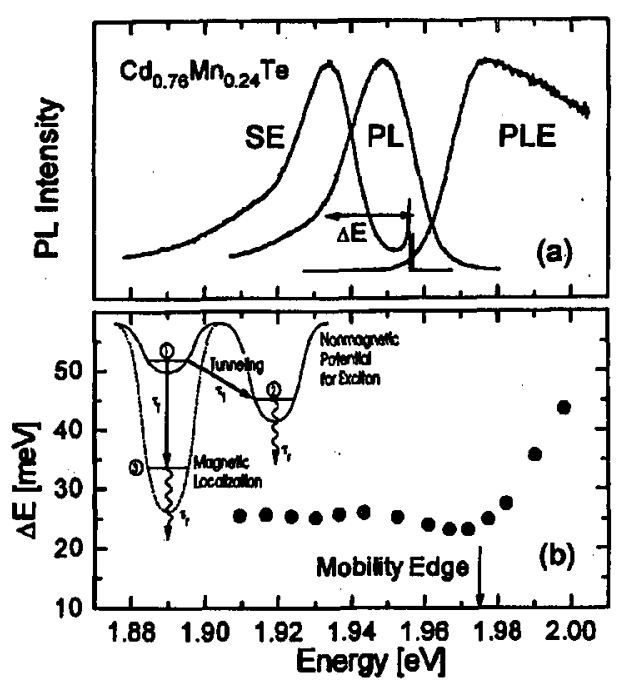

Fig. 1. (a) Spectra of a $\mathrm{Cd}_{0.76} \mathrm{Mn}_{0.24} \mathrm{Te}$ epilayer: photoluminescence spectrum (PL) excited at $\hbar \omega_{\text {exc }}=2.41 \mathrm{eV}$, photoluminescence excitation spectrum (PLE), and spectrum under selective excitation (SE) at $\hbar \omega_{\text {exc }}=1.957 \mathrm{eV} . T=1.8 \mathrm{~K}$. (b) Shift $\Delta E$ (see (a)) as a function of excitation energy. The insert illustrates the competition between nonmagnetic spectral relaxation $(1 \rightarrow 2)$ and magnetic polaron formation $(1 \rightarrow 3)$.

In Fig. 1a we display PL spectra of a $2-\mu \mathrm{m}$ thick $\mathrm{Cd}_{0.76} \mathrm{Mn}_{0.24} \mathrm{Te}$ epilayer grown on CdTe substrate. The PL spectrum was taken under nonselective excitation conditions, i.e. with an excitation energy exceeding the energy of free excitons. The peak is attributed to the recombination of localized excitons. The maximum of the PL excitation spectrum gives the free exciton energy. For selective excitation (SE) in the band of localized states at energies, where the spectral diffusion due to phonon assisted tunneling does not occur during the exciton lifetime, the shift $\Delta E$ between the luminescence line and the excitation energy is due to the magnetic polaron formation only. Thus, the shift $\Delta E$, which is plotted in Fig. 1b as a function of excitation energy, takes on a constant value of $24 \mathrm{meV}$ in the 
regime of localized states and represents the LMP energy [5]. For excitation energies exceeding EME nonmagnetic spectral relaxation occurs and $\Delta E$ varies with the excitation energy. We consider the energy where $\Delta E$ becomes constant as an EME for excitons (see Fig. 1b).

EME is subject to the competition between spectral diffusion and nonmagnetic and magnetic localization. The magnetic polarons are formed with time constants $\left(\tau_{\mathrm{f}}\right)$ of about $100 \mathrm{ps}$, while exciton lifetimes are about $600 \mathrm{ps}$ for $\mathrm{Cd}_{0.76} \mathrm{Mn}_{0.24} \mathrm{Te}[5]$. This rather fast magnetic localization interrupts further spatial diffusion and nonmagnetic spectral relaxation to deeper localization sites $\left(\tau_{\mathrm{t}}\right)$. In the insert of Fig. $1 \mathrm{~b}$ this competition between polaron formation $(1 \rightarrow 3)$ and nonmagnetic spectral relaxation $(1 \rightarrow 2)$ is illustrated. In external magnetic fields the influence of magnetic localization is suppressed and the nonmagnetic one favoured. This is examined in magnetic fields by studying the spectra shown in Fig. 1a.

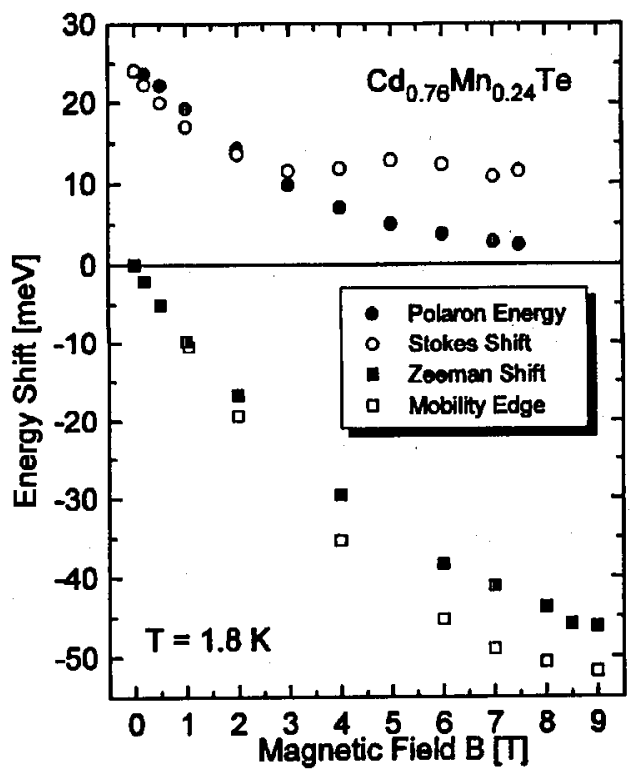

Fig. 2. Polaron energy, Stokes shift, Zeeman shift, and shift of the mobility edge in magnetic fields applied in the Faraday geometry for a $\mathrm{Cd}_{0.76} \mathrm{Mn}_{0.24} \mathrm{Te}$ epilayer.

In Fig. 2 the suppression of the LMP energy is shown (filled circles). A magnetic field of $B=8 \mathrm{~T}$ suppresses the LMP energy to less than $10 \%$ of its zero field value. The free exciton peak in the PL excitation spectrum is Zeeman splitted. Filled squares represent the lower branch taken with $\sigma^{+}$-polarized excitation light. The large Zeeman splitting of the band states prohibits to consider EME in absolute energies. The shift of EME (given by open squares in Fig. 2) has to be taken with respect to the free exciton energy. In magnetic fields $B>3 \mathrm{~T}$ the difference between the Zeeman shift and the shift of EME appears (about $7 \mathrm{meV}$ at $8 \mathrm{~T}$ ). This shows that in magnetic fields EME moves to energies where the 
density of states is lower. In the upper part of Fig. 2 we compare the suppression of the magnetic polaron energy with the suppression of the Stokes shift between PLE and PL (open circles). The PL line is Stokes-shifted with respect to the free exciton energy due to the localization of the excitons. The suppressions of the Stokes shift and the LMP energy are similar for small fields (below $3 \mathrm{~T}$ ) and differ for higher fields. Only the Stokes shift is affected by a shift of EME. The difference between the Stokes shift and the LMP energy of $8 \mathrm{meV}$ at high magnetic fields corresponds well to the shift of EME. Thus, the behaviour of the Stokes shift is explained by taking two contributions into account: the suppression of the LMP formation and the shift of EME, which partly compensate each other for $B>3 \mathrm{~T}$.

In conclusion, we have demonstrated that the effective mobility edge for excitons can be observed in semimagnetic semiconductors due to the additional magnetic localization. We have found the effect of the magnetic polaron formation on the exciton mobility edge. In external magnetic fields the magnetic localization is suppressed and the nonmagnetic spectral relaxation due to phonon emission proceeds deeper into the tail of localized states.

\section{References}

[1] E. Cohen, M.D. Sturge, Phys. Rev. B 25, 3828 (1982).

[2] H.X. Jiang, L.Q. Zu, J.Y. Lin, Phys. Rev. B 42, 7284 (1990).

[3] V. F. Aguekian, S. Takeyama, S. Adachi, Y. Takagi, N. Miura, Solid State Commun. 86, 205 (1993).

[4] A. Golnik, J. Ginter, J.A. Gaj, J. Phys. C 16, 6073 (1983).

[5] G. Mackh, W. Ossau, D.R. Yakovlev, A. Waag, G. Landwehr, R. Hellmann, E.O. Göbel, Phys. Rev. B 49, 10248 (1994).

[6] T. Itoh, E. Komatsu, J. Lumin. 38, 266 (1987). 